Hincapié-Cruz, J.P., \& Márquez, E.J. (2021). Variación fenotípica de los peces Curimata mivartii (Characiformes: Curimatidae) y Pimelodus grosskopfii (Siluriformes: Pimelodidae) en hábitats lóticos y lénticos. Revista de Biología Tropical, 69(2), 434-444. DOI 10.15517/rbt. v69i2.41708

DOI 10.15517/rbt.v69i2.41708

\title{
Variación fenotípica de los peces Curimata mivartii (Characiformes: Curimatidae) y Pimelodus grosskopfii (Siluriformes: Pimelodidae) en hábitats lóticos y lénticos
}

\author{
Juan Pablo Hincapié-Cruz ${ }^{1 *}$ \\ Edna J. Márquez ${ }^{1 * *}$
}

1. Facultad de Ciencias, Universidad Nacional de Colombia, Medellín, Colombia. Calle 59A No 63 - 20 Bloque 19 A Laboratorio 310, Medellín, Colombia; jphincapiec@unal.edu.co, ejmarque@unal.edu.co, ejmarque@gmail.com; *Deceased; ** Correspondence.

\author{
Recibido 05-V-2020. Corregido 26-I-2021. Aceptado 08-II-2021.
}

\author{
ABSTRACT \\ Phenotypic variation of the fishes Curimata mivartii (Characiformes: Curimatidae) \\ and Pimelodus grosskopfii (Siluriformes: Pimelodidae) in lotic and lentic habitats
}

\begin{abstract}
Introduction: Despite their importance in swimming performance and individual fitness, body shape and size variations in response to water flow velocity remain unknown for most migratory freshwater fishes in the Neotropics. Objective: Test the hypothesis of phenotypic variation in the size and body shape of two species of freshwater fishes that encounter changes in water velocity across their migrations in two rivers that belong to a single Neotropical basin. Methods: Landmark-based geometric morphometrics was used to study variations in body size and shape for the fish species Curimata mivartii and Pimelodus grosskopfii in lotic and lentic habitats of the Colombian Magdalena-Cauca Basin. Results: Individuals of C. mivartii from two lotic habitats were similar in size and both were significantly bigger than those from lentic habitats, whereas individuals of $P$. grosskopfii were similar in size in both habitats. Both species showed more streamlined bodies in lotic habitats and deeper body shapes in lentic habitats. Conclusions: Both species exhibit phenotypic changes in body shape concordant with previous predictions on morphological variations of fishes. This information is relevant to predict changes in response to environmental heterogeneity, especially those induced by anthropogenic activities that change the water velocity in the river.
\end{abstract}

Key words: geometric morphometrics; phenotypic variation; phenotypic plasticity; flow regimes.

Los órdenes Characiformes y Siluriformes poseen respectivamente 2258 y 3974 especies de peces válidas, las cuales se encuentran ampliamente distribuidas a lo largo del planeta (Fricke, Eschmeyer, \& Fong, 2020). En Colombia tienen una presencia importante en los diferentes sistemas hídricos en donde se han registrado 644 especies de Characiformes y 588 especies de Siluriformes, que representan respectivamente el 43.1 y $39.3 \%$ de las especies dulceacuícolas del país (DoNascimiento, Herrera Collazos, \& Maldonado-Ocampo, 2019). Dos miembros de estos órdenes, Curimata mivartii (Steindachner, 1878) (Characiformes: Curimatidae) y Pimelodus grosskopfii (Steindachner, 1879) (Siluriformes: Pimelodidae), 
se encuentran entre las 10 especies más desembarcadas en la cuenca Magdalena-Cauca (Servicio Estadístico Pesquero ColombianoSEPEC, 2020) y se han categorizado como peces amenazados, debido a la disminución en la calidad del hábitat y su explotación directa (Jiménez-Segura, 2016; Villa-Navarro, Usma, Mesa-Salazar, \& Sanchez-Duarte, 2016).

Curimata mivartii, conocida como "vizcaína", es una especie detritívora, endémica de la cuenca Colombiana Magdalena-Cauca, recientemente agregada a la lista de especies de los ríos de la vertiente del Caribe (DoNascimiento et al., 2019) y categorizada como casi amenazada (NT) con una vulnerabilidad A2d (Jiménez-Segura, 2016); aun cuando se ha indicado que su rango de migración es corto $(<100$ $\mathrm{km}$ ) (Lasso et al., 2011a; López-Casas, Jiménez-Segura, Agostinho, \& Pérez, 2016), estudios genético-poblacionales recientes indican que puede estar subestimado, considerando el flujo génico observado en un rango de $350 \mathrm{~km}$ (Landínez-García \& Marquez, 2018). Pimelodus grosskopfii, conocido como "capaz", tiene hábitos alimentarios omnívoros con tendencia carnívora y preferencia por insectos, macroinvertebrados y peces (Ramírez \& Pinilla, 2012), es endémico de la cuenca Colombiana del Magdalena-Cauca y está categorizado en peligro crítico (CR) con nivel de vulnerabilidad A2d en la lista roja de la IUCN (Villa-Navarro et al., 2016). Su rango de migración es medio (100-500 km; Usma et al., 2013) y los estudios genéticos de sus poblaciones muestran diferencias genéticas en escalas geográficas grandes en la cuenca del río Magdalena (Villa-Navarro, 2002) y flujo génico en la cuenca del río Cauca (Restrepo-Escobar, Yepes-Acevedo, \& Márquez, 2021).

Las causas de variación fenotípica de estos peces son desconocidas, aun cuando se reconoce que en otras especies, el tamaño y la conformación del cuerpo están influenciados por el componente genético (Hendry, Taylor, \& McPhail, 2002), la ontogenia (Colangelo, Ventura, Piras, Bonaiuti, \& Ardizzone, 2019), el sexo (Hastings, 2019), el gradiente altitudinal (Alarcón-Durán, Castillo-Rivera,
Figueroa-Lucero, Arroyo-Cabrales, \& Barriga-Sosa, 2017), la temperatura (Geladakis, Nikolioudakis, Koumoundouros, \& Somarakis, 2018; Reyes Corral \& Aguirre, 2019), los hábitos alimenticios (Svanbäck \& Eklöv, 2003; Restrepo-Escobar, Hurtado-Alarcón, ManceraRodríguez, \& Márquez, 2016), la presencia o ausencia de depredadores (Gomes \& Monteiro, 2008), así como los regímenes de flujo de agua (Langerhans, 2008; Langerhans \& Reznick, 2010) y las modificaciones antrópicas del hábitat (Santos \& Araújo, 2015; Hamilton, Rolshausen, Uren Webster, \& Tyler, 2017).

En cuanto a los regímenes de flujo, Langerhans (2008) predice que, para flujos rápidos, las formas corporales suelen desarrollarse fusiformes o hidrodinámicas con el fin de obtener un mejor rendimiento en la natación constante, en oposición a una conformación robusta que se presenta en el flujo lento; condición que es más óptima para nadar a velocidad variable (Langerhans, 2008). Se piensa que estos cambios corporales pueden influenciar el desempeño locomotor de los peces, convirtiéndose en un agente evolutivo que actúa en la biodiversidad acuática o reflejan plasticidad fenotípica (Haas, Blum, \& Heins, 2010). Estas predicciones, están soportadas con estudios en los que se compararon entre individuos de lagos y arroyos (Langerhans, Layman, Langerhans, \& Dewitt, 2003; Franssen, 2011; Gaston, \& Lauer, 2015). Cabe resaltar algunas excepciones a las predicciones de Langerhans (2008), las cuales se explican por presiones selectivas, hábitos comportamentales, estrategias de alimentación, entre otros (Hendry et al., 2002; Franssen, Stewart, \& Schaefer, 2013; Meyers \& Belk, 2014).

La evaluación de las predicciones de Langerhans (2008) en C. mivartii y P. grosskopfii, ha sido el objetivo principal del presente estudio. Estas especies constituyen dos historias de vida distintas que coexisten en ambientes con heterogeneidad espacial y temporal en la cuenca Magdalena-Cauca (Lasso et al., 2011a; Lasso et al., 2011b). Ambas especies se exponen a cambios de hábitats y de velocidad del agua durante sus migraciones contracorriente 
desde las ciénagas o planos inundables hacia el canal principal de estos ríos, en la compleja orografía andina (Maldonado-Ocampo et al., 2005). Para contrastar esta hipótesis, se seleccionó la Morfometría Geométrica, metodología en la cual la forma de un organismo se analiza incorporando su geometría, gracias al uso de puntos anatómicos de referencia (en inglés, landmarks) (Rohlf \& Bookstein, 1990), altamente diferenciados y homólogos entre individuos (Zelditch, Swiderski, \& Sheets, 2012). La geometría descrita por dichos puntos, posteriormente se compara con métodos estadísticos univariados y multivariados, los cuales se combinan con herramientas de visualización directa de la forma biológica (Klingenberg, 2016). Dado que el tamaño es una de las variables que describe la forma de un organismo, también evaluamos su variación con el fin estimar su influencia en las variaciones de la conformación del cuerpo de los peces estudiados.

\section{MATERIALES Y MÉTODOS}

Área de estudio y organismos examinados: el área de estudio incluyó hábitats lóticos y lenticos de las cuencas de los ríos Cauca y Magdalena en Colombia: Cauca-Léntico (C-LE; 8 14'48" N - 7443'28' W \& 8०47'32" $\mathrm{N}-7^{\circ} 24^{\prime} 57^{\prime}$ 'W; ciénaga, 25 m.s.n.m., 30 ${ }^{\circ} \mathrm{C}$, velocidad promedio anual de $0 \pm 0.1 \mathrm{~m} / \mathrm{s}$ ); Cauca-Lótico (C-LO; 7¹3'51.712" N \& $75^{\circ} 30^{\prime} 27.691$ ' W; canal principal de río, 150 m.s.n.m., $24{ }^{\circ} \mathrm{C}$, velocidad promedio anual de $1.18 \pm 0.52 \mathrm{~m} / \mathrm{s}$ ) y Magdalena-Lótico (M-LO; $6^{\circ} 30^{\prime} 19^{\prime \prime} \mathrm{N} \& 74^{\circ} 23^{\prime} 40^{\prime \prime} \mathrm{W}$; canal principal del río, 125 m.s.n.m, $27.8^{\circ} \mathrm{C}$, velocidad promedio anual de $0.93 \pm 0.16 \mathrm{~m} / \mathrm{s}$ ). Se ha indicado que los peces migratorios en el río Magdalena utilizan el canal principal para desovar y las ciénagas o planicies de inundación como hábitats de cría (Jiménez-Segura, Palacio, \& Leite, 2010). Las muestras fueron recolectadas durante los períodos migratorios (diciembrefebrero; junio-agosto) en el 2014, 2016 y 2017 utilizando trasmallo y atarraya. En total se analizaron 153 individuos, 106 de C. mivartii (longitud estándar: 13.285-21.137 cm; C-LE:
44; C-LO: 21; M-LO: 41) y 47 de P. grosskopfii (longitud estándar: 10.462-22.861 cm; C-LE: 20; C-LO: 27).

Morfometría geométrica: las fotografías en vista lateral izquierda de los individuos frescos ubicados sobre una superficie plana y utilizando una escala milimétrica, se tomaron con una cámara marca CANON PowerShot SX 130 IS, a una distancia de $33 \mathrm{~cm}$ del objetivo, dejando a los individuos en el centro del campo visual y alejados del margen de la fotografía, con el fin de evitar deformaciones ópticas en su periferia. Se seleccionaron puntos anatómicos de referencia (tipo I: intersección de tejidos; tipo II: máxima curvatura/profundidad) (Bookstein, 1991) que cumplen los criterios de homología, cobertura, distribución, coplanaridad y no redundancia (Klingenberg, 2008; Zelditch et al., 2012). Para C. mivartii (Fig. 1) se identificaron 10 puntos anatómicos de referencia, 9 tipo I y 1 tipo II (punta del hocico); para $P$. grosskopfii (Fig. 2) se identificaron 12 puntos anatómicos de referencia tipo I.

Los módulos del paquete XYOM v.2.0 (Dujardin \& Dujardin, 2019) se utilizaron para la digitalización de las coordenadas de los puntos anatómicos, la repetibilidad de la digitalización de las coordenadas, la generación de variables de tamaño y conformación, así como también los análisis de pendientes alométricas, y análisis discriminante de componentes principales. La cuantificación de la precisión en la digitalización de las coordenadas, se llevó a cabo siguiendo la metodología descrita por Bolaños-Cubillos, Campos y Márquez (2016). Posteriormente, las coordenadas crudas se sometieron al Análisis generalizado de Procrustes, con el fin de obtener las variables de tamaño y conformación, libres de variación no biológica (Rohlf \& Bookstein, 1990; Goodall, 1991).

Análisis estadísticos: como variable de tamaño se utilizó el "tamaño centroide", el cual se define como la raíz cuadrada de la suma de los cuadrados de las distancias, de todos los puntos de referencia a su centroide 


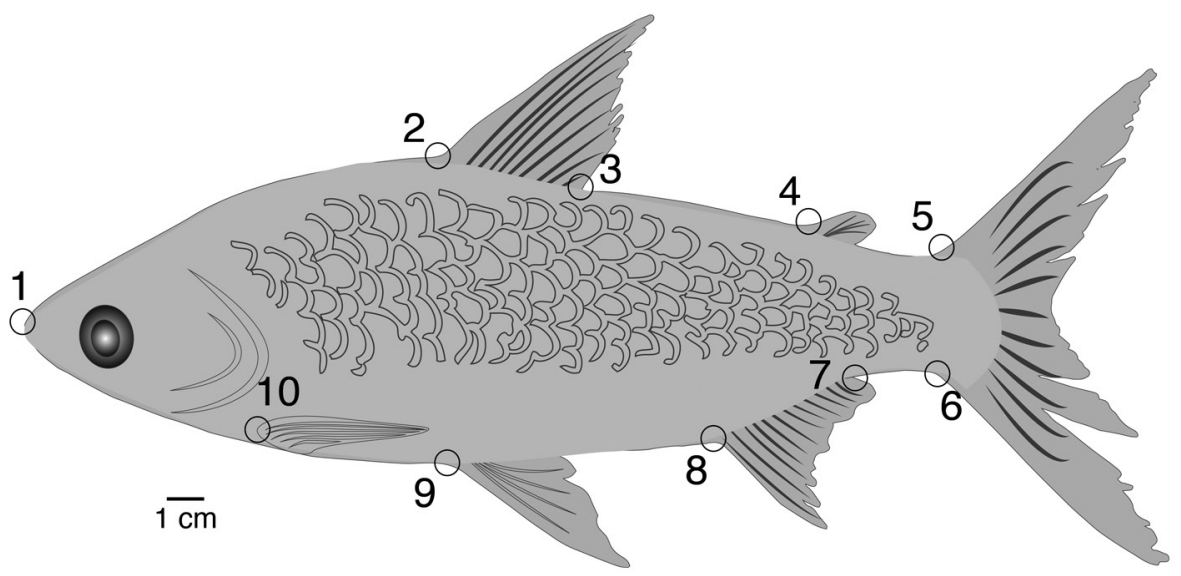

Fig. 1. Puntos anatómicos de referencia usados para el análisis morfométrico de C. mivartii. 1. punta del hocico; 2-3, inserción anterior y posterior de la aleta dorsal; 4, inserción anterior de la aleta adiposa; 5-6, inserción del ultimo rayo dorsal y ultimo rayo ventral de la aleta caudal; 7-8, inserción posterior y anterior de la aleta anal; 9, inserción de la aleta pélvica; 10, inserción de la aleta pectoral (Por Mauricio Urrego Tobón).

Fig. 1. Landmarks used for morphometric analysis of C. mivartii. 1. tip of snout; 2-3, dorsal-fin anterior and posterior insertion, respectively; 4, adipose-fin anterior insertion; 5-6, caudal-fin last dorsal and ventral ray insertion, respectively; 7-8, anal-fin posterior and anterior insertion, respectively; 9, pelvic-fin insertion; 10, pectoral-fin insertion (By Mauricio Urrego Tobón).

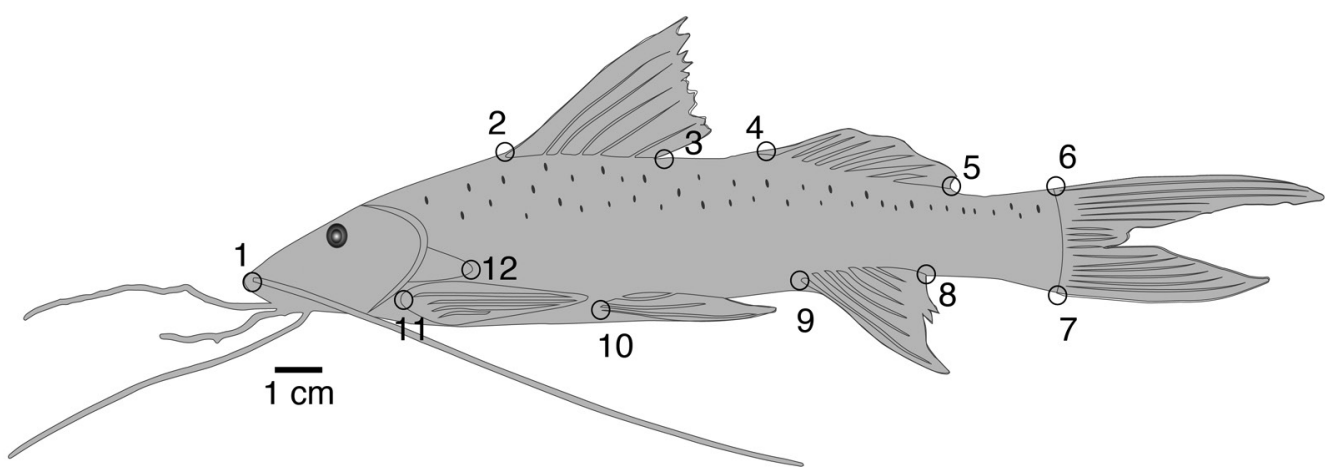

Fig. 2. Puntos anatómicos de referencia usados para el análisis morfométrico de P. grosskopfii. 1, base de la barbilla maxilar; 2-3, inserción anterior y posterior de la aleta dorsal; 4-5, inserción anterior y posterior de la aleta adiposa; 6-7, inserción del ultimo rayo dorsal y ultimo rayo ventral de la aleta caudal; 8-9, inserción posterior y anterior de la aleta anal; 10, inserción de la aleta pélvica; 11, inserción de la aleta pectoral; 12, apófisis cleitral (Por Mauricio Urrego Tobón).

Fig. 2. Landmarks used for morphometric analysis of P. grosskopfii. 1, maxillary barbel base; 2-3, dorsal-fin anterior and posterior insertion, respectively; 4-5, adipose-fin anterior and posterior insertion, respectively; 6-7, caudal-fin last dorsal and ventral ray insertion respectively; 8-9, anal-fin posterior and anterior insertion, respectively; 10, pelvic-fin insertion; 11, pectoral-fin insertion; 12, cleithral apophysis (By Mauricio Urrego Tobón).

(Klingenberg, Barluenga, \& Meyer, 2002). Como variables de conformación se utilizaron los componentes uniformes y no uniformes, los cuales reflejan las deformaciones globales y regionales de cada objeto, respectivamente (Bookstein, 1991). En cada especie, las comparaciones del tamaño entre hábitats se llevaron a cabo utilizando una prueba ANOVA de 1 vía y comparaciones pareadas con las pruebas Tukey, si se satisfacían los supuestos requeridos para aplicar una prueba paramétrica, los cuales fueron evaluados con el programa 
estadístico R v.3.5.3. (R Core Team, 2013). En caso contrario, se utilizaron las pruebas Kruskal Wallis y las comparaciones pareadas de Mann Whitney.

Las comparaciones de la conformación del cuerpo de los individuos en los diferentes hábitats, se llevó a cabo utilizando las distancias euclidianas $(\mathrm{DEu})$ entre contrastes y sus respectivas significancias estadísticas se calcularon con 1000 permutaciones y se ajustaron por Bonferroni en las comparaciones múltiples (Rice, 1989). Para detectar la existencia de alometría (efecto residual del tamaño centroide sobre las variables de conformación) se realizó un análisis de regresión multivariado y su significancia estadística se calculó mediante 1000 permutaciones (Good, 2000). Cuando el efecto alométrico fue significativo, se efectuó un análisis multivariado de covarianza para contrastar la hipótesis de que las pendientes alométricas de los diferentes hábitats tenían un modelo común. En caso positivo, se llevó a cabo una corrección por tamaño y se recalculó la significancia estadística. Además, se realizó un análisis discriminante de componentes principales y un análisis gráfico de placa delgada con el fin de visualizar las deformaciones en las conformaciones de los cuerpos de los peces, en relación con sus hábitats utilizando el programa tpsRegr v.1.45 (Rohlf, 2016).

\section{RESULTADOS}

La repetibilidad en las medidas de las coordenadas estuvo entre 0.891-0.991 para C. mivartii y $0.854-0.993$ para $P$. grosskopfii. Los datos del tamaño centroide cumplieron los supuestos de homocedasticidad en ambas especies; sin embargo, el supuesto de normalidad se cumplió en $P$. grosskopfii pero no en C. mivartii (Tabla 1).

Los individuos de C. mivartii de los ambientes lóticos de los ríos Cauca (promedio: $18.8 \mathrm{~cm}$ ) y Magdalena (promedio: $18.5 \mathrm{~cm}$ ) exhibieron tamaños centroides similares, significativamente más grandes que los del ambiente léntico del Cauca (promedio: $16.2 \mathrm{~cm}$; Tabla 1, Fig. 3). En contraste, los individuos de

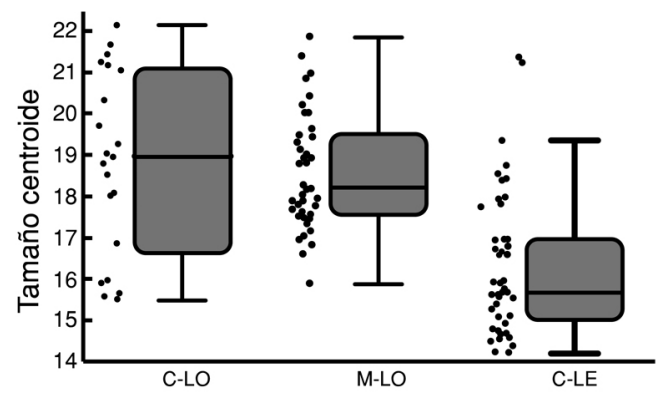

Fig. 3. Variación del tamaño centroide $(\mathrm{cm})$ de $C$. mivartii en los hábitats lótico (C-LO) y léntico (C-LE) del río Cauca y hábitat lótico del río Magdalena (M-LO).

Fig. 3. Size variation $(\mathrm{cm})$ of C. mivartii in lotic (C-LO) and lentic (C-LE) habitats of Cauca River and lotic habitat of Magdalena River (M-LO).

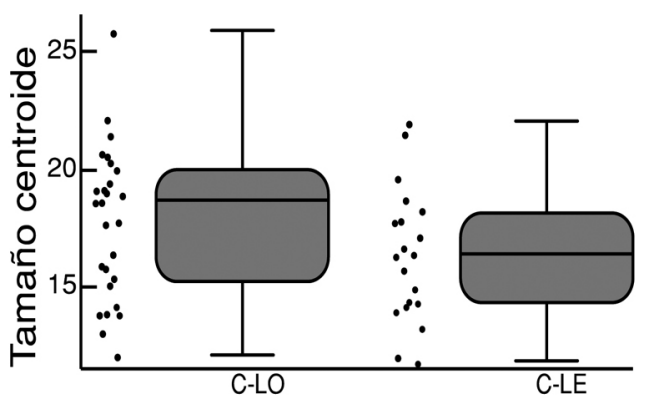

Fig. 4. Variación $(\mathrm{cm})$ del tamaño centroide de $P$. grosskopfii en los hábitats lótico (C-LO) y léntico (C-LE) del río Cauca.

Fig. 4. Size variation (cm) of $P$. grosskopfii in lotic (C-LO) and lentic (C-LE) habitats of the Cauca River.

P. grosskopfii no exhibieron diferencias significativas entre los hábitats lótico $(17.8 \mathrm{~cm})$ y léntico (16.5 cm) del Cauca (Tabla 1, Fig. 4).

En las comparaciones de la conformación entre ambientes lóticos (C-LO vs M-LO) y lénticos (M-LO vs C-LE) que incluyeron al río Magdalena, el efecto alométrico no fue significativo (Tabla 2). Sin embargo, en las comparaciones entre los ambientes lóticos y lénticos del río Cauca (C-LO vs C-LE), el efecto alométrico fue significativo y las pendientes alométricas fueron similares, en ambas especies (Tabla 2).

Entre ambientes lóticos, la conformación del cuerpo de $C$. mivartii fue significativamente más fusiforme en el río Cauca que en el río 
TABLA 1

Pruebas estadísticas para contrastar homocedasticidad, normalidad y diferencias del tamaño centroide de

Curimata mivartii y Pimelodus grosskopfii entre los hábitats lóticos y lénticos de los ríos Cauca y Magdalena

TABLE 1

Statistic tests for homoscedasticity, normality and difference of centroid size of Curimata mivartii and Pimelodus grosskopfii among lotic and lentic habitats in the Cauca and Magdalena rivers

\begin{tabular}{lcc}
\multicolumn{1}{c}{ Prueba } & Estadístico & $\mathrm{P}$ \\
Curimata mivartii & & \\
$\mathrm{F}_{(2,103)}$ & 2.983 & 0.055 \\
Shapiro Wilks & 0.974 & 0.036 \\
$\mathrm{U}(\mathrm{C}-\mathrm{LO}$ vs M-LO) & 383 & 1.000 \\
$\mathrm{U}(\mathrm{M}-\mathrm{LO}$ vs C-LE) & 268 & $<0.001$ \\
$\mathrm{U}(\mathrm{C}-\mathrm{LO}$ vs C-LE) & 170 & $<0.001$ \\
Pimelodus grosskopfii & & \\
$\mathrm{F}_{(1,45)}$ & 0.401 & 0.529 \\
Shapiro Wilks & 0.980 & 0.594 \\
$\mathrm{~F}_{(1,45)}$ (C-LO vs C-LE) & 2.362 & 0.131 \\
\hline
\end{tabular}

C-LO, C-LE: hábitats lóticos y lénticos del Río Cauca, respectivamente; M-LO: hábitat lótico del Río Magdalena; F: Prueba F (grados de libertad del numerador y denominador); U: Prueba U de Mann-Whitney.

C-LO, C-LE: lotic and lentic habitats in the Cauca River, respectively; M-LO: lotic habitat in the Magdalena River; F: F test (numerator and denominator degrees of freedom); U: Mann-Whitney U test.

Magdalena (Tabla 2, Fig. 5). Además, los individuos del sistema lótico del río Cauca exhibieron cuerpos más fusiformes que los del sistema léntico del mismo río (Tabla 2, Fig. 5). De igual manera, los individuos del sistema lótico del río Magdalena exhibieron cuerpos más fusiformes que los del sistema léntico del río Cauca (Tabla 2, Fig. 5). Un comportamiento similar se observó en P. grosskopfii quien exhibió cuerpos más fusiformes en el ambiente lótico que en el léntico del río Cauca (Tabla 2, Fig. 6).

\section{DISCUSIÓN}

El presente trabajo contrastó la hipótesis de diferenciación fenotípica a través de
TABLA 2

Regresión multivariada (Rm), distancias euclidianas (DEu) y significancias estadísticas (P) de la conformación corporal de Curimata mivartii y Pimelodus grosskopfii en los hábitats lóticos y lénticos de los ríos Cauca y Magdalena

TABLE 2

Multivariate regression (Rm), Euclidian distances (DEu) and statistical significance $(\mathrm{P})$ of the body shape of

Curimata mivartii and Pimelodus grosskopfii in the lotic and lentic habitats in the Cauca and Magdalena rivers

\begin{tabular}{lccc}
\multicolumn{1}{c}{ Contraste } & $\mathrm{P}(\mathrm{Rm})$ & $\mathrm{DEu}$ & $\mathrm{P}(\mathrm{DEu})$ \\
Curimata mivartii & & & \\
C-LO vs M-LO & $>0.050$ & 0.012 & 0.012 \\
M-LO vs C-LE & $>0.050$ & 0.014 & 0.021 \\
C-LO vs C-LE & 0.026 & 0.012 & 0.016 \\
C-LO vs C-LE* & & 0.018 & $<0.001$ \\
Pimelodus grosskopfii & & & \\
C-LO vs C-LE & $<0.001$ & 0.024 & $<0.001$ \\
C-LO vs C-LE & & 0.022 & $<0.001$ \\
\hline
\end{tabular}

C-LO, C-LE: hábitats lóticos y lénticos del Río Cauca, respectivamente; M-LO: hábitat lótico del Río Magdalena; $\mathrm{DEu}$ libre de alometría en ${ }^{*} \mathrm{~F}_{(16.46)}=1,255 ; \mathrm{P}=0.266$ and ${ }^{\ddagger} \mathrm{F}_{(20.24)}=1,105 ; \mathrm{P}=0.403$.

C-LO, C-LE: lotic and lentic habitats in the Cauca River, respectively; M-LO: lotic habitat in the Magdalena River; non-allometric $\mathrm{DEu}$ in ${ }^{*} \mathrm{~F}_{(16.46)}=1,255 ; \mathrm{P}=0.266$ and ${ }^{\dagger} \mathrm{F}_{(20.24)}=1,105 ; \mathrm{P}=0.403$.

diferentes regímenes de flujos de agua (Langerhans, 2008), en dos especies de peces neotropicales; uno de los modelos, C. mivartii se suma a las especies evaluadas con morfometría geométrica del orden Characiformes (Santos \& Araújo, 2015; Restrepo-Escobar et al., 2016; Lazzarotto, Barros, Louvise, \& Caramaschi, 2017; de Barros, Louvise, \& Caramaschi, 2019; Rodrigues Rocha, de Mesquita Alves, Pasa, \& Frehner Kavalco, 2019) y el segundo modelo, P. grosskopfii, constituye el primer miembro de Siluriformes evaluado con morfometría geométrica para la determinación de la variación del tamaño y la conformación con respecto a los regímenes de flujo de agua.

En contraste con $P$. grosskopfii que no mostró diferencias significativas entre los diferentes sistemas del río Cauca, los individuos de 


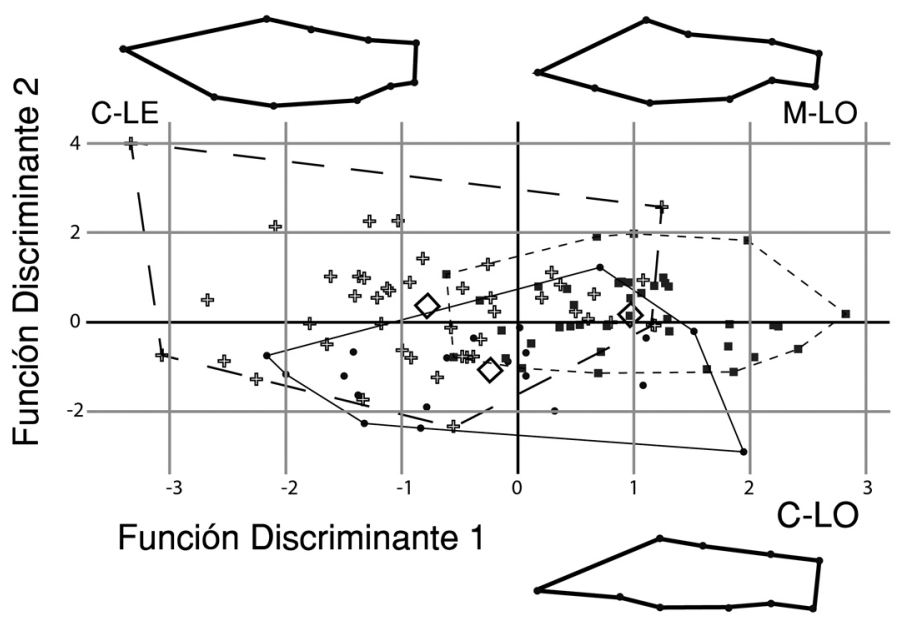

Fig. 5. Diferencias en la conformación del cuerpo de C. mivartii en los hábitats lénticos (C-LE) y lóticos de los ríos Cauca (C-LO) y Magdalena (M-LO). FD1 y FD2: Función discriminante 1 y 2, respectivamente. Rejillas de deformación aumentadas x 10 .

Fig. 5. Differences in the body shape of C. mivartii in lentic (C-LE) and lotic habitats of Cauca (C-LO) and Magdalena (M-LO) rivers. FD1 and FD2: Discriminant function 1 and 2, respectively. Thin-plate spline transformations at x10 magnification.

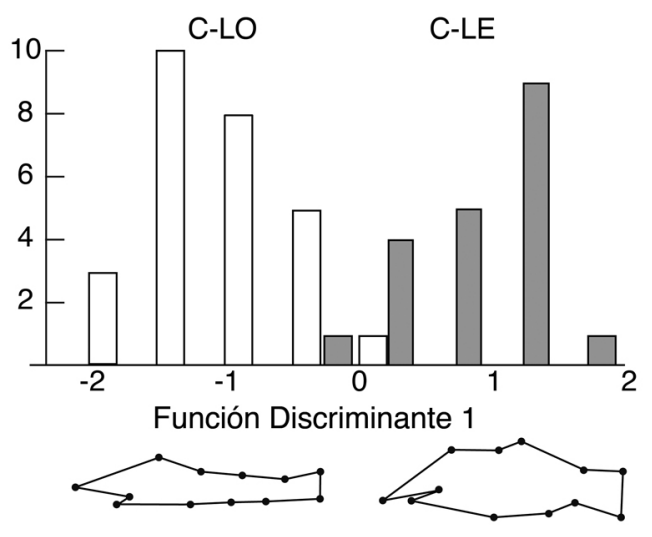

Fig. 6. Diferencias en la conformación del cuerpo de $P$. grosskopfii en los hábitats lótico (C-LO) y léntico (C-LE) del río Cauca representadas en rejillas de deformación aumentadas x 3 .

Fig. 6. Differences in the body shape of $P$. grosskopfii in lotic (C-LO) and lentic (C-LE) habitats of Cauca River represented by thin-plate spline transformations at $\mathrm{x} 3$ magnification.

C. mivartii de los ambientes lóticos de ambos ríos, fueron significativamente más grandes que los del ambiente léntico del río Cauca. Siguiendo a Jiménez-Segura et al., (2010), los resultados de C. mivartii podrían reflejar la separación estacional de los individuos que participan en los eventos de migración/reproducción (ambiente lótico) de los que permanecen en los sitios de crianza (ambiente léntico). En Brycon henni, el comportamiento reproductivo de la especie parece explicar la presencia de individuos más grandes en los cauces de los ríos colombianos Nare y Guatapé, que en sus quebradas asociadas (Restrepo-Escobar et al., 2016). Sin embargo, los resultados de este estudio contrastan con los encontrados en Hyphessobrycon ericae en los ríos del amazonas brasilero, Sacará y Araticum, donde los tamaños de los individuos fueron significativamente menores en ambientes con altas velocidades que en los de baja velocidad (de Barros et al., 2019). Estos hallazgos, en conjunto con los estudios previos, indican que diferentes factores intrínsecos o medioambientales podrían estar influenciando las diferencias de tamaño, lo que estimula investigaciones futuras.

Debido a que las diferencias en tamaño podrían influir en las variaciones de conformación entre grupos, se evaluó el efecto alométrico entre los grupos comparados y se corrigió el efecto del tamaño en los casos en los 
cuales se encontraron pendientes alométricas similares. Para ambas especies se encontraron diferencias significativas libres de alometría, lo que indica que las diferencias observadas entre los ambientes evaluados no pueden atribuirse a diferencias por tamaño.

Ambas especies fueron más fusiformes en los ambientes lóticos que en el léntico, lo cual concuerda con estudios previos (Hendry et al., 2002; Langerhans et al., 2003; McGuigan, Franklin, Moritz, \& Blows, 2003) y apoya la hipótesis que, en flujos rápidos de agua, las conformaciones corporales suelen presentarse fusiformes o hidrodinámicas; mientras que, en el flujo lento, se espera una conformación robusta (Langerhans, 2008). Además, en los sistemas lóticos, los cuerpos de los individuos de $C$. mivartii del río Cauca fueron más fusiformes que los del río Magdalena, lo que concuerda con que el sitio muestreado en el río Magdalena presenta menor velocidad. En esta zona, el río Magdalena alcanza territorios de pendientes menos pronunciadas, además de ensanchamientos que producen cadenas irregulares de ciénagas de poca profundidad, lo cual podría explicar una reducción (Lasso, Gutiérrez, et al. 2011b) o mayor variación en la velocidad del flujo de agua. En este escenario, los peces pueden tolerar flujos rápidos de agua en ciertos períodos y alternar con sitios donde dicho flujo es amortiguado por las características del paisaje.

Concomitante con los cambios fenotípicos en respuesta a la velocidad del agua, los cuerpos fueron más fusiformes a medida que incrementó la altitud. Una tendencia similar relacionada con gradientes altitudinales se encontró en peces del género Rhoadsia en las vertientes occidentales de los andes ecuatorianos (Malato et al., 2017), con altitudes 4 a 7 veces superiores a la máxima elevación examinada en este estudio. En relación inversa con la altitud, los cuerpos más fusiformes se observaron en las temperaturas más bajas, lo cual está en línea con lo observado en otras especies de peces (Loy, Ciccotti, Ferrucci, \& Cataudella, 1996; O’Reilly \& Horn, 2004;
Georgakopoulou et al., 2007; Scott \& Johnston, 2012; Reyes Corral \& Aguirre, 2019).

Además, dado que $P$. grosskopfii (Restrepo-Escobar et al., 2021) y C. mivartii (Landínez-García \& Marquez, 2018) exhiben flujo génico entre los sitios examinados, los cambios fenotípicos observados no pueden atribuirse a diferenciación genética, apoyando la idea de que las muestras genéticamente similares de cada especie exhiben variaciones fenotípicas como posible respuesta a la heterogeneidad ambiental. Tal explicación es factible para estas especies de peces, que deben ajustar su desempeño locomotor a los eventos migratorios asociados a los ciclos hidrológicos bimodales de los ríos Cauca y Magdalena (Jiménez-Segura et al., 2010; Lasso et al., 2011a; Lasso et al., 2011b). Debido a la ausencia de información disponible, queda por explorar el papel de la presencia de depredadores en las variaciones fenotípicas observadas.

La información obtenida en este estudio amplía el conocimiento sobre las respuestas de los peces ante los cambios en los regímenes del agua y sirve de base para predecir cambios futuros en el ensamblaje de peces, de acuerdo con su habilidad para responder a la heterogeneidad ambiental. Este punto es importante debido a que se ha documentado sobre actividades antropogénicas que inducen respuestas morfológicas en el cuerpo de los peces tales como, el embalsamiento de agua (Haas et al., 2010), la urbanización (Kern \& Langerhans, 2019), alteraciones de patrones en el uso del agua y la tierra (Pease, Grabowski, Pease, \& Bean, 2018), cambios en la temperatura y turbulencia del agua (Reyes Corral \& Aguirre, 2019), entre otros.

Declaración de ética: los autores declaran que todos están de acuerdo con esta publicación y que han hecho aportes que justifican su autoría; que no hay conflicto de interés de ningún tipo; y que han cumplido con todos los requisitos y procedimientos éticos y legales pertinentes. Todas las fuentes de financiamiento se detallan plena y claramente en la sección de agradecimientos. El respectivo documento 
legal firmado se encuentra en los archivos de la revista.

\section{AGRADECIMIENTOS}

Los autores agradecen a la empresa consultora Integral S. A., por proporcionar las fotografías y datos de recolección de las muestras examinadas. Este estudio fue financiado por los proyectos CT-2013-002443 "Variación genotípica y fenotípica de poblaciones de especies reófilas presentes en el área de influencia del proyecto hidroeléctrico Ituango" y CT-2019-000661 "Variabilidad genética de un banco de peces de los sectores medio y bajo del Río Cauca".

\section{RESUMEN}

Introducción: A pesar de su importancia en el rendimiento de la natación y la eficacia biológica individual, la forma del cuerpo y las variaciones de tamaño en respuesta a la velocidad del flujo de agua siguen siendo desconocidas para la mayoría de los peces migratorios de agua dulce en el Neotrópico. Objetivo: Contrastar la hipótesis de variación fenotípica en el tamaño y la conformación del cuerpo de dos especies de peces dulceacuícolas que enfrentan cambios de velocidad a través de sus migraciones en dos ríos pertenecientes a una cuenca neotropical. Métodos: Se utilizó la morfometría geométrica basada en hitos para estudiar las variaciones en el tamaño y la conformación del cuerpo para dos especies de peces Curimata mivartii y Pimelodus grosskopfii en hábitats lóticos y lénticos de la cuenca Colombiana del Magdalena-Cauca. Resultados: Los individuos de C. mivartii de dos hábitats lóticos fueron similares en tamaño y significativamente más grandes que los del hábitat léntico, mientras que los individuos de $P$. grosskopfii fueron similares en ambos hábitats. Ambas especies mostraron cuerpos más hidrodinámicos en hábitats lóticos y formas corporales más robustas en hábitats lénticos. Conclusión: Ambas especies exhiben cambios fenotípicos en la forma del cuerpo concordantes con las predicciones previas sobre las variaciones morfológicas de los peces. Esta información es relevante para predecir cambios en respuesta a la heterogeneidad ambiental, especialmente inducidos por actividades antropogénicas que cambian las velocidades del agua en el río.

Palabras clave: morfometría geométrica; variación fenotípica; plasticidad fenotípica; regímenes de flujo.

\section{REFERENCIAS}

Alarcón-Durán, I., Castillo-Rivera, M.A., Figueroa-Lucero, G., Arroyo-Cabrales, J., \& Barriga-Sosa, I. (2017). Diversidad morfológica en 6 poblaciones del pescado blanco Chirostoma humboldtianum. Revista Mexicana de Biodiversidad, 88(1), 207-214.

Bolaños-Cubillos, N., Campos, N.H., \& Márquez, E.J. (2016). Variación fenotípica de la langosta espinosa Panulirus argus (Decapoda: Paniluridae) en el Caribe Suroccidental. Revista de Biología Tropical, 64(3), 975-990.

Bookstein, F.L. (1991). Morphometric tools for landmark data. New York, USA: Cambridge University Press.

Colangelo, P., Ventura, D., Piras, P., Bonaiuti, J.P.G., \& Ardizzone, G. (2019). Are developmental shifts the main driver of phenotypic evolution in Diplodus spp. (Perciformes: Sparidae)? BMC Evolutionary Biology, 19(1), 106-118.

de Barros, T.F., Louvise, J., \& Caramaschi, É.P. (2019). Flow gradient drives morphological divergence in an Amazon pelagic stream fish. Hydrobiologia, 833(1), 217-229.

DoNascimiento, C., Herrera Collazos, E.E., \& MaldonadoOcampo, J.A. (2019). Lista de especies de peces de agua dulce de Colombia/Checklist of the freshwater fishes of Colombia. Version 2.11, Bogotá, Colombia. Asociación Colombiana de Ictiólogos. Dataset/Checklist. DOI: $10.15472 /$ numrso

Dujardin, S., \& Dujardin, J.P. (2019). Geometric morphometrics in the cloud. Infection, Genetics and Evolution, 70, 189-196.

Franssen, N.R. (2011). Anthropogenic habitat alteration induces rapid morphological divergence in a native stream fish. Evolutionary Applications, 4(6), 791-804.

Franssen, N.R., Stewart, L.K., \& Schaefer, J.F. (2013). Morphological divergence and flow-induced phenotypic plasticity in a native fish from anthropogenically altered stream habitats. Ecology and Evolution, 3(14), 4648-4657.

Fricke, R., Eschmeyer, W.N., \& Fong, J.D. (2020). Catalog of fishes: species by family/subfamily, San Francisco, California, USA. Retrieved from: http://researcharchive.calacademy.org/research/ichthyology/catalog/ SpeciesByFamily.asp Electron version accessed 05 May 2020.

Gaston, K.A., \& Lauer, T.E. (2015). Morphometric variation in bluegill Lepomis macrochirus and green sunfish Lepomis cyanellus in lentic and lotic systems. Journal of Fish Biology, 86(1), 317-332. 
Geladakis, G., Nikolioudakis, N., Koumoundouros, G., \& Somarakis, S. (2018). Morphometric discrimination of pelagic fish stocks challenged by variation in body condition. ICES Journal of Marine Science, 75(2), 711-718.

Georgakopoulou, E., Sfakianakis, D.G., Kouttouki, S., Divanach, P., Kentouri, M., \& Koumoundouros, G. (2007). The influence of temperature during early life on phenotypic expression at later ontogenetic stages in sea bass. Journal of Fish Biology, 70(1), 278-291.

Gomes, J.L., \& Monteiro, L.R. (2008). Morphological divergence patterns among populations of Poecilia vivipara (Teleostei Poeciliidae): test of an ecomorphological paradigm. Biological Journal of the Linnean Society, 93(4), 799-812.

Good, P. (2000). Permutation tests: a practical guide to resampling methods for testing hypotheses. New York, USA: Springer.

Goodall, C. (1991). Procrustes methods in the statistical analysis of shape. Journal of the Royal Statistical Society: Series B (Methodological), 53(2), 285-321.

Haas, T.C., Blum, M.J., \& Heins, D.C. (2010). Morphological responses of a stream fish to water impoundment. Biology Letters, 6(6), 803-806.

Hamilton, P.B., Rolshausen, G., Uren Webster, T.M., \& Tyler, C.R. (2017). Adaptive capabilities and fitness consequences associated with pollution exposure in fish. Philosophical Transactions of the Royal Society B: Biological Sciences, 372(1712), 20160042.

Hastings, P.A. (2019). Evolution of sexual dimorphism in tube blennies (Teleostei: Chaenopsidae). Integrative Organismal Biology, 1(1), obz003.

Hendry, A.P., Taylor, E.B., \& McPhail, J.D. (2002). Adaptive divergence and the balance between selection and gene flow: lake and stream stickleback in the Misty system. Evolution, 56(6), 1199-1216.

Jiménez-Segura, L. (2016). Curimata mivartii. The IUCN Red List of Threatened Species 2016: e.T167634A61472671. DOI: 10.2305/IUCN. UK.2016-1.RLTS.T167634A61472671.en

Jiménez-Segura, L.F., Palacio, J., \& Leite, R. (2010). River flooding and reproduction of migratory fish species in the Magdalena River basin, Colombia. Ecology of Freshwater Fish, 19(2), 178-186.

Kern, E., \& Langerhans, R.B. (2019). Urbanization alters swimming performance of a stream fish. Frontiers in Ecology and Evolution, 6, 229-241.

Klingenberg, C.P. (2008). Novelty and "homology-free" morphometrics: what's in a name? Evolutionary Biology, 35(3), 186-190.
Klingenberg, C.P. (2016). Size, shape, and form: concepts of allometry in geometric morphometrics. Development Genes and Evolution, 226(3), 113-137.

Klingenberg, C.P., Barluenga, M., \& Meyer, A. (2002). Shape analysis of symmetric structures: quantifying variation among individuals and asymmetry. Evolution, 56(10), 1909-1920.

Landínez-García, R.M., \& Marquez, E.J. (2018). Microsatellite loci development and population genetics in Neotropical fish Curimata mivartii (Characiformes: Curimatidae). PeerJ, 6, e5959.

Langerhans, R.B. (2008). Predictability of phenotypic differentiation across flow regimes in fishes. Integrative and Comparative Biology, 48(6), 750-768.

Langerhans, R.B., Layman, C.A., Langerhans, A.K., \& Dewitt, T.J. (2003). Habitat-associated morphological divergence in two Neotropical fish species. Biological Journal of the Linnean Society, 80(4), 689-698.

Langerhans, R.B., \& Reznick, D.N. (2010). Ecology and evolution of swimming performance in fishes: predicting evolution with biomechanics. Fish Locomotion: An Eco-ethological Perspective, 220, 248.

Lasso, C.A., Agudelo-Córdoba, E., Jiménez-Segura, L.F., Ramírez-Gil, H., Morales-Betancourt, M.A., AjiacoMartínez, R.E., ... Sanabria Ochoa, A.I. (2011a). Catálogo de los recursos pesqueros continentales de Colombia: memoria técnica y explicativa, resumen ejecutivo. Bogotá D.C., Colombia: Instituto de Investigación de Recursos Biológicos Alexander von Humboldt.

Lasso, C.A., Morales-Betancourt, M.A., González-Cañón, G., Ajiaco-Martínez, R.E., Valderrama Barco, M., Hernández Barrero, S., ... Bonilla-Castillo, C.A. (2011b). II. Pesquerias continentales de Colombia: cuencas del Magdalena-Cauca, Sinú, Canalete, Atrato, Orinoco, Amazonas y vertiente del Pacífico. Bogotá D.C., Colombia: Instituto de Investigación de Recursos Biológicos Alexander von Humboldt.

Lazzarotto, H., Barros, T., Louvise, J., \& Caramaschi, É.P. (2017). Morphological variation among populations of Hemigrammus coeruleus (Characiformes: Characidae) in a Negro River tributary, Brazilian Amazon. Neotropical Ichthyology, 15(1), e160152.

López-Casas, S., Jiménez-Segura, L.F., Agostinho, A.A., \& Pérez, C.M. (2016). Potamodromous migrations in the Magdalena River basin: bimodal reproductive patterns in neotropical rivers. Journal of Fish Biology, 89(1), 157-171.

Loy, A., Ciccotti, E., Ferrucci, L., \& Cataudella, S. (1996). An application of automated feature extraction and geometric morphometrics: temperature-related changes in body form of Cyprinus carpio juveniles. Aquacultural Engineering, 15(4), 301-311. 
Malato, G., Shervette, V.R., Navarrete Amaya, R., Valdiviezo Rivera, J., Nugra Salazar, F., Calle Delgado, P., ... Aguirre, W.E. (2017). Parallel body shape divergence in the Neotropical fish genus Rhoadsia (Teleostei: Characidae) along elevational gradients of the western slopes of the Ecuadorian Andes. PloS One, 12(6), e0179432.

Maldonado-Ocampo, J.A., Ortega-Lara, A., Usma, J.S., Galvis, G., Villa-Navarro, F.A., Vásquez, L., ... Ardila, C. (2005). Peces de los Andes de Colombia. Bogotá, Colombia: Instituto Humboldt Colombia.

McGuigan, K., Franklin, C.E., Moritz, C., \& Blows, M.W. (2003). Adaptation of rainbow fish to lake and stream habitats. Evolution, 57(1), 104-118.

Meyers, P.J., \& Belk, M.C. (2014). Shape variation in a benthic stream fish across flow regimes. Hydrobiologia, 738(1), 147-154.

O'Reilly, K.M., \& Horn, M.H. (2004). Phenotypic variation among populations of Atherinops affinis (Atherinopsidae) with insights from a geometric morphometric analysis. Journal of Fish Biology, 64(4), 1117-1135.

Pease, J.E., Grabowski, T.B., Pease, A.A., \& Bean, P.T (2018). Changing environmental gradients over forty years alter ecomorphological variation in Guadalupe Bass Micropterus treculii throughout a river basin. Ecology and Evolution, 8(16), 8508-8522.

Ramírez, A., \& Pinilla, G. (2012). Trophic habits, morphometry and gonadal status of five fish species in different climatic periods at the Río Sogamoso (Santander, Colombia). Acta Biológica Colombiana, 17(2), 241-258.

R Core Team. (2013). $R$ : A language and environment for statistical computing. $\mathrm{R}$ foundation for Statistical Computing, Viena, Austria. Retrieved from: http// www.R-project.org

Restrepo-Escobar, N., Hurtado-Alarcón, J.C., ManceraRodríguez, N.J., \& Márquez, E.J. (2016). Variations of body geometry in Brycon henni (Teleostei: Characiformes, Bryconidae) in different rivers and streams. Journal of Fish Biology, 89(1), 522-528.

Restrepo-Escobar, N., Yepes-Acevedo, A.J., \& Márquez, E.J. (2021). Microsatellite loci development for three catfish species from Northwestern South America. Neotropical Ichthyology, 19(1): e200040. https://doi. org/10.1590/1982-0224-2020-0040

Reyes Corral, W.D., \& Aguirre, W.E. (2019). Effects of temperature and water turbulence on vertebral number and body shape in Astyanax mexicanus (Teleostei: Characidae). PloS One, 14(7), e0219677.

Rice, W.R. (1989). Analyzing tables of statistical tests. Evolution, 43(1), 223-225

Rodrigues Rocha, R., de Mesquita Alves, R., Pasa, R., \& Frehner Kavalco, K. (2019). Morphological and genetic structure of two equivalent Astyanax species (Characiformes: Characidae) in the region of Paranaíba Arc. The Scientific World Journal, 2019, 1-8.

Rohlf, F.J., \& Bookstein, F.L. (1990). Proceedings of the Michigan morphometrics workshop. Michigan, USA: University of Michigan Museum of Zoology.

Rohlf, F.J. (2016). tpsRegr, shape regression, version 1.45. Department of Ecology and Evolution, State University of New York at Stony Brook, USA. Retrieved from: http://www.sbmorphometrics.org/index.html

Santos, A.B.I., \& Araújo, F.G. (2015). Evidence of morphological differences between Astyanax bimaculatus (Actinopterygii: Characidae) from reaches above and below dams on a tropical river. Environmental Biology of Fishes, 98(1), 183-191.

Scott, G.R., \& Johnston, I.A. (2012). Temperature during embryonic development has persistent effects on thermal acclimation capacity in zebrafish. Proceedings of the National Academy of Sciences, 109(35), 14247-14252.

Servicio Estadístico Pesquero Colombiano-SEPEC (2020). Pesca de consumo. Autoridad Nacional de Acuicultura y Pesca, Bogotá, Colombia. Retrieved from: http:// sepec.aunap.gov.co

Steindachner, F. (1878). Zur Fischfauna des MagdalenenStromes. Anzeiger der Akademie der Wissenschaften in Wien, 15, 88-91.

Steindachner, F. (1879). Ichthyologische Beiträge (VIII). Anzeiger der Akademie der Wissenschaften in Wien, 16, 194-195.

Svanbäck, R., \& Eklöv, P. (2003). Morphology dependent foraging efficiency in perch: a trade-off for ecological specialization? Oikos, 102(2), 273-284.

Usma-Oviedo, S., Villa-Navarro, F., Lasso, C.A., Castro, F., Zuñiga, P. T., Cipamocha, C.A., ... Maldonado-Ocampo, J.A. (2013). Peces dulceacuícolas migratorios. Guía de las especies migratorias de la biodiversidad en Colombia, 2, 216-485.

Villa-Navarro, F.A. (2002). Diferenciación entre poblaciones de Pimelodus clarias y Pimelodus grosskopfii (Siluriformes: Pimelodidae) en la cuenca del río Magdalena (Colombia). Cali, Colombia: Universidad del Valle.

Villa-Navarro, F.A., Usma, J.S., Mesa-Salazar, L., \& Sanchez-Duarte, P. (2016). Pimelodus grosskopfii. The IUCN Red List of Threatened Species 2016: e.T49829828A61473588. DOI: 10.2305/IUCN. UK.2016-1.RLTS.T49829828A61473588.en

Zelditch, M.L., Swiderski, D.L., \& Sheets, H.D. (2012). Geometric morphometrics for biologists: a primer. New York, USA: Academic Press. 